\title{
THE PRIME GEODESIC THEOREM IN SQUARE MEAN
}

\author{
ANTAL BALOG, ANDRÁS BIRÓ, GERGELY HARCOS, AND PÉTER MAGA
}

\begin{abstract}
We strengthen the recent result of Cherubini-Guerreiro on the square mean of the error term in the prime geodesic theorem for $\mathrm{PSL}_{2}(\mathbb{Z})$. We also develop a short interval version of this result.
\end{abstract}

\section{INTRODUCTION}

The aim of this note is to provide a new upper bound for the square mean error in the classical prime geodesic theorem. For a brief introduction, let $\Gamma:=\mathrm{PSL}_{2}(\mathbb{Z})$ be the modular group, and let

$$
\Psi_{\Gamma}(X):=\sum_{N P \leqslant X} \Lambda(P)
$$

denote the usual Chebyshev-like counting function for the closed geodesics on the modular surface $\Gamma \backslash \mathcal{H}$. That is, $\log N P$ is the length of the closed geodesic $P$, and $\Lambda(P)=\log N P_{0}$ is the length of the underlying prime closed geodesic $P_{0}$. The closed geodesic $P$ (resp. $P_{0}$ ) is understood without orientation, hence it corresponds bijectively to an unordered pair of hyperbolic (resp. primitive hyperbolic) conjugacy classes in $\Gamma$ which are reciprocals of each other (cf. [Sa1, Sa2]). In an original breakthrough, Iwaniec [Iw1] proved that

$$
\Psi_{\Gamma}(X)=X+O_{\varepsilon}\left(X^{35 / 48+\varepsilon}\right)
$$

for any $\varepsilon>0$, the important point being that 35/48 in the exponent is less than $3 / 4$. This constant was subsequently lowered to $7 / 10$ by Luo-Sarnak [LuSa], $71 / 102$ by Cai [Ca], and $25 / 36$ by SoundararajanYoung $[\mathrm{SoYo}]$. For the last mentioned result, Balkanova-Frolenkov $[\mathrm{BaFr}]$ provided a new proof very recently. It is conjectured that the exponent $2 / 3+\varepsilon$ or perhaps even $1 / 2+\varepsilon$ is admissible (in which case it would be optimal). Our main result states that the exponent $7 / 12+\varepsilon$ is valid in a square mean sense.

Theorem 1. Let $A>2$. Then, for any $\varepsilon>0$ we have

$$
\frac{1}{A} \int_{A}^{2 A}\left|\Psi_{\Gamma}(X)-X\right|^{2} d X \ll_{\varepsilon} A^{7 / 6+\varepsilon} .
$$

This estimate improves on the result of Cherubini-Guerreiro [ChGu, Th. 1.4], where the right hand side was $A^{5 / 4+\varepsilon}$, and in fact our analysis is based on theirs. Incidentally, the exponents $7 / 12+\varepsilon$ and $5 / 8+\varepsilon$ also occur in the recent works of Petridis-Risager [PeRi] and Biró [Bi] on the hyperbolic circle problem, although their averages are not fully analogous to ours.

Theorem 1 has the following simple consequence for short intervals. For $0 \leqslant \eta \leqslant 1$ we have

$$
\frac{1}{A} \int_{A}^{2 A}\left|\Psi_{\Gamma}(X)-\Psi_{\Gamma}(X-\eta X)-\eta X\right|^{2} d X \ll_{\varepsilon} A^{7 / 6+\varepsilon},
$$

that is, the approximation $\Psi_{\Gamma}(X)-\Psi_{\Gamma}(X-\eta X) \approx \eta X$ is valid with error term $X^{7 / 12+\varepsilon}$ in a square mean sense. For $\eta \geqslant A^{-1 / 6}$, this is the best we can say at the moment. However, for smaller $\eta$, we can obtain an improvement by tailoring our analysis to the present problem, with an average error term tending to $X^{1 / 2+\varepsilon}$ as $\eta$ gets close to $A^{-1 / 2}$.

2010 Mathematics Subject Classification. Primary 11F72; Secondary 11M36.

Key words and phrases. Prime geodesic theorem, spectral exponential sums.

Supported by NKFIH (National Research, Development and Innovation Office) grant K 119528 and by the MTA Rényi Intézet Lendület Automorphic Research Group. Fourth author also supported by the Premium Postdoctoral Fellowship of the Hungarian Academy of Sciences. 
Theorem 2. Let $A>2$. Then, for any $\varepsilon>0$ and $A^{-1 / 2} \log ^{2} A \leqslant \eta<A^{-1 / 6}$ we have

$$
\frac{1}{A} \int_{A}^{2 A}\left|\Psi_{\Gamma}(X)-\Psi_{\Gamma}(X-\eta X)-\eta X\right|^{2} d X \ll_{\varepsilon} A^{5 / 4+\varepsilon} \eta^{1 / 2}
$$

Remark 1. Theorem 2 can be improved for very small $\eta$ by employing [BaFr, Th. 8.3]. Specifically, on the right hand side of the bound, $A^{1+\varepsilon}$ is admissible for $A^{-1 / 2} \log ^{2} A \leqslant \eta<A^{-4 / 9}$, and $A^{5 / 3+\varepsilon} \eta^{3 / 2}$ is admissible for $A^{-4 / 9} \leqslant \eta<A^{-5 / 12}$. See also Remark 2 below Theorem 3 .

The paper is structured as follows. The overall strategy is already present in Iwaniec's seminal paper [Iw1], but we also rely crucially on the work of Cherubini-Guerreiro [ChGu] and Luo-Sarnak [LuSa]. In Section 4, we reduce Theorems 1 and 2 to the estimation of a certain spectral exponential sum. This reduction ultimately follows from Selberg's trace formula, although we do not invoke it explicitly. In Section 3, we prove Theorem 3, which contains the necessary bounds for the spectral exponential sum. This proof is ultimately an application of Kuznetsov's trace formula, which again remains in the background, however. Section 2 prepares the scene, incorporating a key idea of Iwaniec [Iw1].

\section{Reduction to KuZnetsov's trace Formula}

Let $\left\{u_{j}\right\}$ be an orthonormal Hecke eigenbasis of the space of Maass cusp forms on $\Gamma \backslash \mathcal{H}$. Denoting by $1 / 4+t_{j}^{2}$ the Laplace eigenvalue of $u_{j}$ with the sign convention $t_{j}>0$, we have the Fourier decomposition

$$
u_{j}(x+i y)=\sqrt{y} \sum_{n \neq 0} \rho_{j}(n) K_{i t_{j}}(2 \pi|n| y) e(n x) .
$$

The Fourier coefficients $\rho_{j}(n)$ are proportional to the Hecke eigenvalues $\lambda_{j}(n)$,

$$
\rho_{j}(n)=\rho_{j}(1) \lambda_{j}(n) .
$$

The Hecke eigenvalues are real, and they satisfy the multiplicativity relations

$$
\lambda_{j}(m) \lambda_{j}(n)=\sum_{d \mid \operatorname{gcd}(m, n)} \lambda_{j}\left(\frac{m n}{d^{2}}\right) .
$$

In particular, the symmetric square $L$-function of $u_{j}$ satisfies

$$
L\left(s, \operatorname{sym}^{2} u_{j}\right)=\zeta(2 s) \sum_{n=1}^{\infty} \frac{\lambda_{j}\left(n^{2}\right)}{n^{s}}=\frac{\zeta(2 s)}{\zeta(s)} \sum_{n=1}^{\infty} \frac{\lambda_{j}(n)^{2}}{n^{s}}, \quad \Re s>1,
$$

in the region of absolute convergence of both Dirichlet series.

Concerning the distribution of Laplace eigenvalues, we record Weyl's law as (cf. [Iw2, (11.5)])

$$
\#\left\{j: t_{j} \leqslant T\right\}=\frac{T^{2}}{12}+O(T \log T) .
$$

In fact a finer asymptotic expansion is available, see [He, Ch. 11, (2.12)] or [Ve, Th. 7.3].

With Kuznetsov's trace formula in mind, we introduce the harmonic weights

$$
\alpha_{j}:=\frac{\left|\rho_{j}(1)\right|^{2}}{\cosh \left(\pi t_{j}\right)}=\frac{2}{L\left(1, \operatorname{sym}^{2} u_{j}\right)},
$$

which by [Iw2, Th. 8.3] and [HoLo, Th. 0.2] satisfy the convenient bounds

$$
t_{j}^{-\varepsilon} \ll_{\varepsilon} \alpha_{j} \ll_{\varepsilon} t_{j}^{\varepsilon} .
$$

For arbitrary $X, T>2$, we borrow from [DeIw, Lemma 7] the test function (see also [LuSa, p. 234] and [BaFr, Lemma 2.2])

$$
\varphi(x):=\frac{\sinh \beta}{\pi} x \exp (i x \cosh \beta) \quad \text { with } \quad \beta:=\frac{\log X}{2}+\frac{i}{2 T},
$$

whose Bessel transform

$$
\hat{\varphi}(t):=\frac{\pi i}{2 \sinh (\pi t)} \int_{0}^{\infty}\left(J_{2 i t}(x)-J_{-2 i t}(x)\right) \varphi(x) \frac{d x}{x}
$$


satisfies

$$
\hat{\varphi}(t)=\frac{\sinh (\pi t+2 \beta i t)}{\sinh (\pi t)}=X^{i t} e^{-t / T}+O\left(e^{-\pi t}\right) .
$$

Following [Iw1, LuSa], we consider the spectral-arithmetic average (cf. (2))

$$
\sum_{j} \alpha_{j} \hat{\varphi}\left(t_{j}\right) \sum_{n} h(n) \lambda_{j}(n)^{2}=\sum_{j} \alpha_{j} \hat{\varphi}\left(t_{j}\right) \frac{1}{2 \pi i} \int_{(2)} \tilde{h}(s) \frac{\zeta(s)}{\zeta(2 s)} L\left(s, \operatorname{sym}^{2} u_{j}\right) d s,
$$

where $h:(0, \infty) \rightarrow \mathbb{R}$ is a smooth compactly supported function with holomorphic Mellin transform $\tilde{h}$ : $\mathbb{C} \rightarrow \mathbb{C}$. We choose $h$ such that it is supported in some dyadic interval $[N, 2 N]$ for $N>1$, and it satisfies $h^{(j)} \ll_{j} N^{-j}$ and $\tilde{h}(1)=N$. Then also

$$
\tilde{h}(s)=\frac{(-1)^{j}}{s(s+1) \ldots(s+j-1)} \int_{0}^{\infty} h^{(j)}(x) x^{s+j} \frac{d x}{x} \ll_{\sigma, j} \frac{N^{\sigma}}{(1+|s|)^{j}}, \quad \Re(s)=\sigma,
$$

where the implied constant depends continuously on $\sigma$. More precisely, the identity is meant for $s$ outside $\{0,-1,-2, \ldots\}$, but the inequality holds even at these exceptional points. Shifting the contour, we obtain by the residue theorem and (4),

$$
\sum_{j} \alpha_{j} \hat{\varphi}\left(t_{j}\right) \sum_{n} h(n) \lambda_{j}(n)^{2}=\frac{12 N}{\pi^{2}} \sum_{j} \hat{\varphi}\left(t_{j}\right)+\sum_{j} \alpha_{j} \hat{\varphi}\left(t_{j}\right) \frac{1}{2 \pi i} \int_{(1 / 2)} \tilde{h}(s) \frac{\zeta(s)}{\zeta(2 s)} L\left(s, \operatorname{sym}^{2} u_{j}\right) d s .
$$

Using also the approximation (6), we obtain after some rearrangement,

$$
\begin{aligned}
\sum_{j} X^{i t_{j}} e^{-t_{j} / T}= & O(1)+\frac{\pi^{2}}{12 N} \sum_{n} h(n) \sum_{j} \alpha_{j} \hat{\varphi}\left(t_{j}\right) \lambda_{j}(n)^{2} \\
& -\frac{\pi^{2}}{12 N} \frac{1}{2 \pi i} \int_{(1 / 2)} \tilde{h}(s) \frac{\zeta(s)}{\zeta(2 s)} \sum_{j} \alpha_{j} \hat{\varphi}\left(t_{j}\right) L\left(s, \operatorname{sym}^{2} u_{j}\right) d s .
\end{aligned}
$$

This formula is equivalent to [BaFr, (3.8)], and we have included the proof for the sake of completeness. We stress that the spectral weights $\hat{\varphi}\left(t_{j}\right)$ depend on the parameters $X, T>2$.

\section{Spectral exponential sums in square mean}

We shall estimate the spectral exponential sum (8), in square mean over $A \leqslant X \leqslant 2 A$, by combining (8) with the analysis of Cherubini-Guerreiro $[\mathrm{ChGu}]$ and Luo-Sarnak [LuSa]. Specifically, on the right hand side of (8), the square mean of the first $j$-sum can be estimated via Kuznetsov's formula and the HardyLittlewood-Pólya inequality (cf. [ChGu, Lemma 4.2]), while the square mean of the second $j$-sum can be estimated in terms of the spectral second moment of symmetric square $L$-functions (cf. [LuSa, (33)]). This way we obtain the following improvement of [ChGu, Prop. 4.5].

Theorem 3. Let $A>2$. Then, for any $\varepsilon>0$ we have

$$
\frac{1}{A} \int_{A}^{2 A}\left|\sum_{t_{j} \leqslant T} X^{i t_{j}}\right|^{2} d X \ll_{\varepsilon}(A T)^{\varepsilon} \begin{cases}T^{3}, & 0<T \leqslant A^{1 / 6} \\ A^{1 / 4} T^{3 / 2}, & A^{1 / 6}<T \leqslant A^{1 / 2} \\ T^{2}, & A^{1 / 2}<T .\end{cases}
$$

In particular, the left hand side can always be bounded as $\ll_{\varepsilon}(A T)^{\varepsilon} A^{1 / 6} T^{2}$.

Remark 2. Theorem 3 can be refined in the medium range by employing [BaFr, Th. 8.3]. Specifically, $A^{1 / 4} T^{3 / 2}$ can be improved to $A^{1 / 2+\theta} T^{1 / 2}$ for $A^{1 / 4+\theta}<T \leqslant A^{1 / 3+2 \theta / 3}$, and to $T^{2}$ for $A^{1 / 3+2 \theta / 3}<T \leqslant A^{1 / 2}$. Note that for $\theta$ any value exceeding $1 / 6$ is admissible by the celebrated work of Conrey-Iwaniec [CoIw, Cor. 1.5]. 
Following the proof of [ChGu, Prop. 4.5], which is based on [LuSa, pp. 235-236], we see that (9) can be deduced from the following smoothened variant, itself a strengthening of [ChGu, Lemma 4.4]:

$$
\frac{1}{A} \int_{A}^{2 A}\left|\sum_{j} X^{i t_{j}} e^{-t_{j} / T}\right|^{2} d X \ll_{\varepsilon}(A T)^{\varepsilon} \begin{cases}T^{3}, & 0<T \leqslant A^{1 / 6} \\ A^{1 / 4} T^{3 / 2}, & A^{1 / 6}<T \leqslant A^{1 / 2} \\ T^{2}, & A^{1 / 2}<T\end{cases}
$$

We shall assume here that $T>2$, since otherwise (10) is trivial. As a first step for the proof of (10), we change in (8) the second occurrence of $\hat{\varphi}\left(t_{j}\right)$ to $X^{i t_{j}} e^{-t_{j} / T}$, and we restrict the integration to $|\Im(s)| \leqslant T^{\varepsilon}$. The error resulting from this change is $O_{\varepsilon}(1)$ by $(7)$ and standard bounds for the symmetric square $L$-function and the Riemann zeta function. Then, applying the Cauchy-Schwarz inequality multiple times and standard bounds for the Riemann zeta function, we arrive at

$$
\begin{aligned}
& \left|\sum_{j} X^{i t_{j}} e^{-t_{j} / T}\right|^{2} \ll_{\varepsilon} 1+\frac{1}{N} \sum_{N \leqslant n \leqslant 2 N}\left|\sum_{j} \alpha_{j} \hat{\varphi}\left(t_{j}\right) \lambda_{j}(n)^{2}\right|^{2} \\
& +\frac{T^{\varepsilon}}{N} \int_{-T^{\varepsilon}}^{T^{\varepsilon}}\left|\sum_{j} \alpha_{j} X^{i t_{j}} e^{-t_{j} / T} L\left(1 / 2+i \tau, \operatorname{sym}^{2} u_{j}\right)\right|^{2} d \tau .
\end{aligned}
$$

We abbreviate

$$
L_{j}(\tau):=L\left(1 / 2+i \tau, \operatorname{sym}^{2} u_{j}\right)
$$

and we average over $A \leqslant X \leqslant 2 A$. Applying [ChGu, Lemma 4.2 $]^{1}$ for the contribution of the $n$-sum on the right hand side, we obtain

$$
\begin{aligned}
& \frac{1}{A} \int_{A}^{2 A}\left|\sum_{j} X^{i t_{j}} e^{-t_{j} / T}\right|^{2} d X \ll_{\varepsilon}\left(N A^{1 / 2}+T^{2}\right)(A N T)^{\varepsilon} \\
& +\frac{T^{\varepsilon}}{N} \int_{-T^{\varepsilon}}^{T^{\varepsilon}} \frac{1}{A} \int_{A}^{2 A}\left|\sum_{j} \alpha_{j} X^{i t_{j}} e^{-t_{j} / T} L_{j}(\tau)\right|^{2} d X d \tau .
\end{aligned}
$$

We apply the Cauchy-Schwarz inequality one more time to facilitate the upcoming analysis. Specifically, we distribute the spectral parameters $t_{j}$ on the right hand side into intervals of length $T$, and this way we get

$$
\left|\sum_{j} \alpha_{j} X^{i t_{j}} e^{-t_{j} / T} L_{j}(\tau)\right|^{2} \ll \sum_{m=1}^{\infty} m^{2}\left|\sum_{(m-1) T \leqslant t_{j}<m T} \alpha_{j} X^{i t_{j}} e^{-t_{j} / T} L_{j}(\tau)\right|^{2} .
$$

Therefore, with the notation

$$
I(T, A, m, \tau):=\frac{1}{A} \int_{A}^{2 A}\left|\sum_{(m-1) T \leqslant t_{j}<m T} \alpha_{j} X^{i t_{j}} e^{-t_{j} / T} L_{j}(\tau)\right|^{2} d X
$$

we infer

$$
\frac{1}{A} \int_{A}^{2 A}\left|\sum_{j} X^{i t_{j}} e^{-t_{j} / T}\right|^{2} d X \ll_{\varepsilon}\left(N A^{1 / 2}+T^{2}\right)(A N T)^{\varepsilon}+\frac{T^{\varepsilon}}{N} \sup _{|\tau| \leqslant T^{\varepsilon}} \sum_{m=1}^{\infty} m^{2} I(T, A, m, \tau) .
$$

\footnotetext{
${ }^{1}$ The definitions of $\nu_{j}(n)$ and $\hat{\phi}(t)$ in [ChGu, LuSa] are slightly in error, in particular their $\rho_{j}(n)=\nu_{j}(n) \cosh \left(\pi t_{j} / 2\right) \operatorname{should}$ really be $\rho_{j}(n)=\nu_{j}(n) \cosh \left(\pi t_{j}\right)^{1 / 2}$. With this correction, $\left|\nu_{j}(n)\right|^{2}$ in [ChGu, LuSa] agrees with our $\alpha_{j} \lambda_{j}(n)^{2}$, thanks to $(1)$ and (4). For precise versions of the relevant Kuznetsov formula, see [Ku, Th. 2] and [Iw2, Th. 9.5].
} 
We bound $I(T, A, m, \tau)$ by squaring out the $j$-sum in (11), then integrating explicitly in $X$, and finally using (5) for $\alpha_{j}$ :

$$
\begin{aligned}
I(T, A, m, \tau) & \ll_{\varepsilon} T^{\varepsilon} e^{-2 m} \sum_{(m-1) T \leqslant t_{j}, t_{k}<m T} \frac{\left.\mid L_{j}(\tau) L_{k}(\tau)\right) \mid}{1+\left|t_{j}-t_{k}\right|} \\
& \leqslant \frac{T^{\varepsilon} e^{-2 m}}{2} \sum_{(m-1) T \leqslant t_{j}, t_{k}<m T} \frac{\left|L_{j}(\tau)\right|^{2}+\left|L_{k}(\tau)\right|^{2}}{1+\left|t_{j}-t_{k}\right|} \\
& =T^{\varepsilon} e^{-2 m} \sum_{(m-1) T \leqslant t_{j}<m T}\left|L_{j}(\tau)\right|^{2} \sum_{(m-1) T \leqslant t_{k}<m T} \frac{1}{1+\left|t_{j}-t_{k}\right|} .
\end{aligned}
$$

By the Weyl law (3), the last $k$-sum is

$$
\sum_{(m-1) T \leqslant t_{k}<m T} \frac{1}{1+\left|t_{j}-t_{k}\right|} \leqslant \sum_{\ell=1}^{\lceil T\rceil} \frac{1}{\ell} \sum_{\substack{(m-1) T \leqslant t_{k}<m T \\ \ell-1 \leqslant\left|t_{j}-t_{k}\right|<\ell}} 1 \ll_{\varepsilon}(m T)^{1+\varepsilon} \sum_{\ell=1}^{\lceil T\rceil} \frac{1}{\ell} \ll_{\varepsilon}(m T)^{1+2 \varepsilon},
$$

whence

$$
I(T, A, m, \tau) \ll_{\varepsilon}(m T)^{1+\varepsilon} e^{-2 m} \sum_{(m-1) T \leqslant t_{j}<m T}\left|L_{j}(\tau)\right|^{2} .
$$

For the last sum, we apply the spectral second moment bound of Luo-Sarnak [LuSa, (33)], obtaining

$$
I(T, A, m, \tau) \ll_{\varepsilon}(m T)^{3+\varepsilon}(1+|\tau|)^{5+\varepsilon} e^{-2 m} .
$$

In combination with (12), this yields

$$
\frac{1}{A} \int_{A}^{2 A}\left|\sum_{j} X^{i t_{j}} e^{-t_{j} / T}\right|^{2} d X \ll_{\varepsilon}\left(N A^{1 / 2}+T^{2}\right)(A N T)^{\varepsilon}+\frac{T^{3+\varepsilon}}{N} .
$$

The last bound improves on the display before [ChGu, Prop. 4.5] in that we have $T^{3+\varepsilon}$ in place of $T^{4+\varepsilon}$. We optimize by setting $N:=A^{-1 / 4} T^{3 / 2}$, which exceeds 1 if and only if $T>A^{1 / 6}$. Assuming this, we obtain (10) readily. For $T \leqslant A^{1 / 6}$ we estimate the left hand side of (10) more directly but along the same ideas. Specifically, let us distribute the spectral parameters $t_{j}$ into intervals of length $T$ as before, apply the Cauchy-Schwarz inequality for the resulting $m$-sum, square out the various $j$-subsums, integrate explicitly in $X$, and then apply the Weyl law (3). We obtain (cf. (13))

$$
\begin{aligned}
\frac{1}{A} \int_{A}^{2 A}\left|\sum_{j} X^{i t_{j}} e^{-t_{j} / T}\right|^{2} d X & \ll \sum_{m=1}^{\infty} m^{2} e^{-2 m} \sum_{(m-1) T \leqslant t_{j}, t_{k}<m T} \frac{1}{1+\left|t_{j}-t_{k}\right|} \\
& \ll_{\varepsilon} \sum_{m=1}^{\infty} m^{2} e^{-2 m}\left(m^{1+\varepsilon} T^{2}\right)(m T)^{1+\varepsilon} \ll_{\varepsilon} T^{3+\varepsilon},
\end{aligned}
$$

which is equivalent to (10) for $T \leqslant A^{1 / 6}$. The proof of Theorem 3 is complete.

\section{Prime geodesic ERror terms in square mean}

In this section, we deduce Theorem 1 and 2 from Theorem 3. For both theorems, we shall assume (without loss of generality) that $A>100$.

Our deduction of Theorem 1 follows almost verbatim the argument of Cherubini-Guerreiro right after the proof of [ChGu, Prop. 4.5]. We reproduce this argument (with small corrections), because we shall use certain steps from it in the proof of Theorem 2. Our starting point is the explicit formula for $\Psi_{\Gamma}(X)$ established by Iwaniec [Iw1, Lemma 1],

$$
\Psi_{\Gamma}(X)=X+\sum_{\left|t_{j}\right| \leqslant T} \frac{X^{1 / 2+i t_{j}}}{1 / 2+i t_{j}}+O\left(\frac{X}{T} \log ^{2} X\right), \quad 2<T \leqslant \frac{X^{1 / 2}}{\log ^{2} X} .
$$


Here the notation $\left|t_{j}\right| \leqslant T$ means that the sum runs through the spectral parameters $\pm t_{j}$ with $t_{j} \leqslant T$ (recall our sign convention $t_{j}>0$ ). With the notation

$$
R(X, T):=\sum_{t_{j} \leqslant T} X^{i t_{j}}
$$

the spectral sum in the explicit formula can be expressed as twice the real part of

$$
\sum_{t_{j} \leqslant T} \frac{X^{1 / 2+i t_{j}}}{1 / 2+i t_{j}}=X^{1 / 2} \frac{R(X, T)}{1 / 2+i T}+i X^{1 / 2} \int_{1}^{T} \frac{R(X, U)}{(1 / 2+i U)^{2}} d U
$$

We specify $T:=A^{1 / 2} / \log ^{2} A$, and we note that $T \leqslant X^{1 / 2} / \log ^{2} X$ holds for any $X \geqslant A$ by the assumption $A>100$. Applying the Cauchy-Schwarz inequality several times,

$$
\begin{aligned}
\frac{1}{A} \int_{A}^{2 A}\left|\sum_{t_{j} \leqslant T} \frac{X^{1 / 2+i t_{j}}}{1 / 2+i t_{j}}\right|^{2} d X & \ll \int_{A}^{2 A}\left|\frac{R(X, T)}{1 / 2+i T}\right|^{2} d X+\int_{A}^{2 A}\left|\int_{1}^{T} \frac{R(X, U)}{(1 / 2+i U)^{2}} d U\right|^{2} d X \\
& \ll \frac{1}{T^{2}} \int_{A}^{2 A}|R(X, T)|^{2} d X+\log T \int_{1}^{T}\left(\int_{A}^{2 A}|R(X, U)|^{2} d X\right) \frac{d U}{U^{3}} .
\end{aligned}
$$

On the right hand side, the first term is $O_{\varepsilon}\left(A^{1+\varepsilon}\right)$ and the second term is $O_{\varepsilon}\left(A^{7 / 6+\varepsilon}\right)$ by Theorem 3. Noting also that the error term in (14) is $O_{\varepsilon}\left(A^{1 / 2+\varepsilon}\right)$, we obtain the bound in Theorem 1.

Now we prove Theorem 2. We specify $T:=A^{1 / 2} / \log ^{2} A$ as before. The condition $A^{-1 / 2} \log ^{2} A \leqslant \eta<$ $A^{-1 / 6}$ then yields $T^{-1} \leqslant \eta<1 / 2$. By the explicit formula (14),

$$
\Psi_{\Gamma}(X)-\Psi_{\Gamma}(X-\eta X)-\eta X=\sum_{\left|t_{j}\right| \leqslant T} X^{1 / 2+i t_{j}} \frac{1-(1-\eta)^{1 / 2+i t_{j}}}{1 / 2+i t_{j}}+O_{\varepsilon}\left(A^{1 / 2+\varepsilon}\right),
$$

and we need to estimate the square mean of this expression over $A \leqslant X \leqslant 2 A$. It suffices to do this with the restriction $t_{j}>0$ on the right hand side, since the original sum over $\left|t_{j}\right| \leqslant T$ is twice the real part of the new sum over $t_{j} \leqslant T$. The contribution of the spectral parameters $t_{j} \leqslant 1 / \eta$ can be rewritten and bounded by the Cauchy-Schwarz inequality as

$$
\frac{1}{A} \int_{A}^{2 A}\left|\int_{1-\eta}^{1}\left(\frac{X}{\xi}\right)^{1 / 2} R(X \xi, 1 / \eta) d \xi\right|^{2} d X \leqslant\left(\int_{1-\eta}^{1} \frac{d \xi}{\xi}\right)\left(\int_{1-\eta}^{1} \int_{A}^{2 A}|R(X \xi, 1 / \eta)|^{2} d X d \xi\right) .
$$

The $X$-integral is $O_{\varepsilon}\left(A^{5 / 4+\varepsilon} \eta^{-3 / 2}\right)$ by Theorem 3, hence the right hand side is $O_{\varepsilon}\left(A^{5 / 4+\varepsilon} \eta^{1 / 2}\right)$. The contribution of the spectral parameters $1 / \eta<t_{j} \leqslant T$ is bounded by

$$
\frac{1}{A} \int_{A}^{2 A}\left|\sum_{1 / \eta<t_{j} \leqslant T} \frac{X^{1 / 2+i t_{j}}}{1 / 2+i t_{j}}\right|^{2} d X+\frac{1}{B} \int_{B}^{2 B}\left|\sum_{1 / \eta<t_{j} \leqslant T} \frac{X^{1 / 2+i t_{j}}}{1 / 2+i t_{j}}\right|^{2} d X
$$

where $B$ abbreviates $(1-\eta) A$. These integrals are very similar to the one we encountered in the proof of Theorem 1, so we can be brief. The first integral in (15) can be bounded by partial summation, the Cauchy-Schwarz inequality, and Theorem 3 as

$$
\begin{aligned}
& \ll \eta^{2} \int_{A}^{2 A}|R(X, 1 / \eta)|^{2} d X+\frac{1}{T^{2}} \int_{A}^{2 A}|R(X, T)|^{2} d X+\log (\eta T) \int_{1 / \eta}^{T}\left(\int_{A}^{2 A}|R(X, U)|^{2} d X\right) \frac{d U}{U^{3}} \\
& \ll_{\varepsilon} A^{5 / 4+\varepsilon} \eta^{1 / 2}+A^{1+\varepsilon}+A^{5 / 4+\varepsilon} \int_{1 / \eta}^{T} U^{-3 / 2} d U \ll A^{5 / 4+\varepsilon} \eta^{1 / 2} .
\end{aligned}
$$

Similarly, the second integral in (15) is $O_{\varepsilon}\left(B^{5 / 4+\varepsilon} \eta^{1 / 2}\right)$, hence also $O_{\varepsilon}\left(A^{5 / 4+\varepsilon} \eta^{1 / 2}\right)$. Finally, the contribution of the error term in (14) is $O_{\varepsilon}\left(A^{1+\varepsilon}\right)$. The proof of Theorem 2 is complete. 


\section{REFERENCES}

[BaFr] O. Balkanova, D. Frolenkov, Bounds for the spectral exponential sum, arXiv: 1803.04201

[Bi] A. Biró, Local average of the hyperbolic circle problem for Fuchsian groups, Mathematika 64 (2018), $159-183$.

[Ca] Y. Cai, Prime geodesic theorem, J. Théor. Nombres Bordeaux 14 (2002), 59-72.

[ChGu] G. Cherubini, J. Guerreiro, Mean square in the prime geodesic theorem, Algebra Number Theory, to appear, arXiv: 1702.00297

[CoIw] J. B. Conrey, H. Iwaniec, The cubic moment of central values of automorphic L-functions, Ann. of Math. 151 (2000), 1175-1216.

[DeIw] J.-M. Deshouillers, H. Iwaniec, The non-vanishing of Rankin-Selberg zeta-functions at special points, In: The Selberg trace formula and related topics (Brunswick, Maine, 1984), 51-95, Contemp. Math., Vol. 53, Amer. Math. Soc., Providence, RI, 1986.

[He] D. A. Hejhal, The Selberg trace formula for PSL(2, R), Vol. 2, Lecture Notes in Mathematics, Vol. 1001, Springer-Verlag, Berlin, 1983.

[HoLo] J. Hoffstein, P. Lockhart, Coefficients of Maass forms and the Siegel zero, With an appendix by D. Goldfeld, J. Hoffstein and D. Lieman, Ann. of Math. 140 (1994), 161-181.

[Iw1] H. Iwaniec, Prime geodesic theorem, J. Reine Angew. Math. 349 (1984), 136-159.

[Iw2] H. Iwaniec, Spectral methods of automorphic forms, 2nd edition, Graduate Studies in Mathematics, Vol. 53, Amer. Math. Soc., Providence, RI, 2002.

[Ku] N. V. Kuznetsov, Petersson's conjecture for cusp of weight zero and Linnik's conjecture. Sums of Kloosterman sums, Math USSR Sbornik 39 (1981), 299-342.

[LuSa] W. Luo, P. Sarnak, Quantum ergodicity of eigenfunctions on $\mathrm{PSL}_{2}(\mathbf{Z}) \backslash \mathbf{H}^{2}$, Inst. Hautes Études Sci. Publ. Math. 81 (1995), 207-237.

[PeRi] Y. Petridis, M. Risager, Local average in hyperbolic lattice point counting, With an appendix by N. Laaksonen, Math. Z. 285 (2017), 1319-1344.

[Sa1] P. Sarnak, Class numbers of indefinite binary quadratic forms, J. Number Theory 15 (1982), $229-247$.

[Sa2] P. Sarnak, Reciprocal geodesics, In: Analytic number theory, 217-237, Clay Math. Proc., Vol. 7, Amer. Math. Soc., Providence, RI, 2007.

[SoYo] K. Soundararajan, M. Young, The prime geodesic theorem, J. Reine Angew. Math. 676 (2013), $105-120$.

[Ve] A. B. Venkov, Spectral theory of automorphic functions and its applications, Translated from the Russian by N. B. Lebedinskaya, Mathematics and its Applications (Soviet Series), Vol. 51, Kluwer Academic Publishers Group, Dordrecht, 1990.

Alfréd Rényi Institute of Mathematics, Hungarian Academy of Sciences, POB 127, Budapest H-1364, Hungary

E-mail address: balog@renyi.hu, biroand@renyi.hu,gharcos@renyi.hu, magapeter@gmail.com

MTA RÉnyi Intézet Lendület Automorphic Research Group

E-mail address: balog@renyi.hu, biroand@renyi.hu, gharcos@renyi.hu, magapeter@gmail.com

Central European University, Nador u. 9, Budapest H-1051, Hungary

E-mail address: harcosg@ceu.edu 